\title{
Superconductivity due to Condensation of Monopoles around RCD Strings in SU(2) Gauge Theory
}

\author{
B. S. Rajput ${ }^{1}$ and Sandeep Kumar ${ }^{2}$ \\ ${ }^{1}$ Department of Physics, Kumaon University, Nainital, India \\ ${ }^{2}$ Department of Physics, D. S. College, Aligarh (U.P), India
}

Correspondence should be addressed to B. S. Rajput, bsrajp@gmail.com

Received 17 September 2010; Revised 24 November 2010; Accepted 1 December 2010

Academic Editor: George Siopsis

Copyright (C) 2010 B. S. Rajput and S. Kumar. This is an open access article distributed under the Creative Commons Attribution License, which permits unrestricted use, distribution, and reproduction in any medium, provided the original work is properly cited.

\begin{abstract}
The study of the condensation of monopoles and the resulting chromomagnetic superconductivity have been undertaken in restricted chromodynamics of SU(2) gauge theory. Constructing the RCD Lagrangian and the partition function for monopoles in terms of string action and the action of the current around the strings, the monopole current in RCD chromo magnetic superconductor has been derived and it has shown that in London' limit the penetration length governs the monopole density around RCD string in chromo magnetic superconductors while with finite (nonzero) coherence length the leading behavior of the monopole density at large distances from the string is controlled by the coherence length and not by the penetration length.
\end{abstract}

\section{Introduction}

Quantum chromo dynamics (QCD) is the most favored color gauge theory of strong interaction whereas superconductivity is a remarkable manifestation of quantum mechanics on a truly macroscopic scale. In the process of current understanding of superconductivity, Rajput [1,2] and Kumar et al. [3, 4] have conceived its hopeful analogy with QCD and demonstrated that the essential features of superconductivity, that is, the Meissner effect and flux quantization, provided the vivid models [5-9] for actual confinement mechanism in QCD. The original ideas explaining color confinement in terms of dual superconductivity of $Q C D$ vacuum were proposed by ' $t$ Hooft and Mandelstam in the series of papers where 't Hooft demonstrated $[7,10,11]$ that the vacuum of gluodynamics behaves as dual superconductor and the key role in dual superconductor model of QCD is played by Abelian monopoles and Mandelstam [12-14] propounded that the color confinement properties may 
result from the condensation of magnetic monopoles in QCD vacuum. In a series of papers [15-18] Ezawa and Iwazaki made an attempt to analyze a mechanism of quark confinement by demonstrating that the Yang-Mills vacuum is magnetic superconductor and such a superconducting state is considered to be a condensed state of magnetic monopole. The condensation of magnetic monopole incorporates the state of magnetic superconductivity [19] and the notion of chromo magnetic superconductor where the Meissner effect, confining magnetic field in ordinary superconductivity, would be replaced by the chromoelectric Meissner effect (i.e., the dual Meissner effect) which would confine the color electric flux. As such one conceives the idea of correspondence between quantum chromo dynamic situation and chromomagnetic superconductor. However, the crucial ingredient for condensation in a chromo magnetic superconductor would be the nonAbelian force in contrast to the Abelian ones in ordinary superconductivity. Topologically, a nonAbelian gauge theory is equivalent to a set of Abelian gauge theories supplemented by monopoles [11].The method of Abelian projection is one of the popular approaches to confinement problem, together with dual superconductivity $[20,21]$ picture, in nonAbelian gauge theories. Monopole condensation mechanism of confinement (together with dual superconductivity) implies that long-range physics is dominated by Abelian degrees of freedom [21] (Abelian dominance).

Evaluating Wilson loops under the influence of the Abelian field due to all monopole currents, monopole dominance has been demonstrated [22, 23]. In the Abelian projection the quarks are the electrically charged particles and, if monopoles are condensed, the dual Abrikasove string carrying electric flux is formed between quark and antiquark. Due to nonzero tension in this string, the quarks are confined by the linear potential. The conjecture that the dual Meissner effect is the color confinement mechanism is realized if we perform Abelian projection in the maximal gauge where the Abelian component of gluon field and Abelian monopoles are found to be dominant [24, 25]. Then the Abelian electric field is squeezed by solenoidal monopole current [26]. The vacuum of gluodynamics behaves as a dual superconductor and the key role in dual superconductor model of QCD is played by Abelian monopole. Therefore an important problem, before studying the vacuum properties of nonAbelian theories, is to abelianize them so as to make contribution of the topological magnetic degrees of freedom to the partition function explicit. To meet this end, a dual gauge theory called restricted chromo dynamics (RCD) (i.e., an Abelian version of nonAbelian QCD) has been constructed out of QCD in SU(2) theory [27-30] by imposing an additional internal symmetry named magnetic symmetry [31-35] which reduces the dynamical degrees of freedom. Attempts have been made [1-4] to establish an analogy between superconductivity and the dynamical breaking of magnetic symmetry, which incorporates the confinement phase in RCD vacuum.

In the present paper this structure of RCD has been used to undertake the study of condensation of monopoles and the resultant chromomagnetic superconductivity in SU(2) gauge theory. The RCD Lagrangian density for monopoles has been derived in magnetic gauge and the resulting partition function has been computed in terms of string action and the action of current around the strings. Using this partition function, the quantum average of Wilson loop for monopoles has been computed and the sources of electric flux (i.e., quarks) running along the trajectory have been introduced with the help of Wilson loop.

The monopole current in RCD chromo magnetic superconductor has been derived in London limit which corresponds to infinitely deep Higgs potential leading to vanishing coherence length. It has been shown that the squared monopole current in RCD chromo magnetic superconductor in the London limit has a maximum at the distance of the order of penetration length and it (the penetration length) governs the monopole density around the 
string in RCD chromomagnetic superconductor. The monopole current has also been derived in RCD chromo magnetic superconductor with nonzero finite coherence length and it has been shown that the monopole density is nonzero even in the absence of string. It has also been shown that the quantum correction to the squared monopole density is much more than its vacuum expectation value measured far outside the string. It has been demonstrated that in the chromo magnetic superconductors with finite (nonzero) coherence length the quantum corrections to squared monopole density control the leading behavior of the total monopole density in the vicinity of the RCD string. It has also been shown that the leading behavior of the monopole density at large distances from the string is controlled by the coherence length and not by the penetration length.

\section{Superconductivity due to Condensation of Monopoles in SU(2) Gauge Theory}

Monopole condensation mechanism of confinement, together with dual superconductivity, implies that long-range physics is dominated by Abelian degrees of freedom and the method of Abelian projection (i.e., Abelianization) is one of the popular approaches to the problem of confinement, and hence superconductivity, in nonAbelian gauge theories. Such an Abelianization of QCD may be obtained in the form of restricted chromodynamics (RCD) by imposing an additional internal symmetry named magnetic symmetry [31-35] which reduces the dynamical degrees of freedom. Mathematical foundation of restricted chromodynamics (RCD) is based on the fact that a nonAbelian gauge theory permits some additional internal symmetry, that is, magnetic symmetry [36-39]. Unified space $P$ of nonAbelian gauge theory may be thought of as

$$
P=M \otimes G
$$

which is $(4+n)$ dimensional manifold where $M$ is 4-dimensional external space and $G$, in general, is the $n$-dimensional internal space, generated by $n$ Killing vectors $\xi_{i}$ satisfying the conditions

$$
\begin{gathered}
{\left[\xi_{i}, \xi_{j}\right]=f_{i j}^{k} \xi_{k},} \\
£_{\xi \xi} g_{A B}=0,
\end{gathered}
$$

where $g_{A B}(A, B=0, \ldots, n+3)$ is the metric of manifold $P$ with gauge symmetry as $n$ dimensional isometry $[40,41]$ and $£_{\xi i}$ is the Lie derivative along $\xi_{i}$. In (2.2) $f_{i j}^{k}$ is internal structure parameter, the four-dimensional quotient space $M=P / G$ is the base manifold and $P$ is the principal fiber bundle. It has been conjectured that the dynamics of magnetic monopole is effectively described by a gauge theory based on magnetic symmetry which has the topological meaning. This magnetic symmetry is an additional internal isometry $H$ having some additional Killing vector fields of generalized gauge theory. These additional Killing vectors are purely internal ones and hence commute with already existing fields $\xi_{i}$ 
of $G$. The internal isometry $H$ is Cartan's subgroup of $G$ and commutes with it. Let the additional Killing vector fields be $m_{a}(a=1,2, \ldots, k=\operatorname{dim} H)$. Then we have

$$
\begin{gathered}
m_{a}=m_{a}^{i} \xi_{i}, \quad(i=1,2,3), \\
\left(\xi_{i}, m_{a}\right)=0 \\
\left(m_{a}, m_{b}\right)=-f_{a b}^{(H)_{c}} m_{(c)} \\
£_{m a} g_{A B}=0
\end{gathered}
$$

where $£_{m a}$ is the Lie derivative along the direction of magnetic symmetry. Since the isometry $H$ commutes with the right isometry $G$, it is called the left isometry. The topological magnetic charge associated with monopoles corresponds to the elements of second homotopy group $\pi_{2}(G / H)$.

Let us consider $G$ as $\mathrm{SU}(2)$ Yang-Mills group and $H$ as the global gauge group of electromagnetic interaction $U(1)$. Then

$$
\pi_{2}\left(\frac{G}{H}\right)=\pi_{2}\left(\frac{\mathrm{SU}(2)}{U(1)}\right)
$$

is determined by the second homotopy directly leading to the magnetic symmetry gauge group of monopoles in terms of $m$. As such, monopoles are described in terms of the topological charges. This magnetic symmetry obviously imposes a strong constraint on the connection and hence may be regarded as symmetry of gauge potential. This gauge symmetry restricts not only the metric but also the gauge potential. Such a restricted theory RCD may be extracted from full QCD on restricting the dynamical degrees of freedom of theory, keeping full gauge degrees of freedom intact, by imposing magnetic symmetry which ultimately forces the generalized nonAbelian gauge potential $V_{\mu}=A_{\mu}-B_{\mu}$, (with $A_{\mu}$ and $B_{\mu}$ as electric and magnetic constituents) to satisfy a strong constraint given by

$$
D_{\mu} \widehat{m}=\partial_{\mu} \widehat{m}+i g \vec{V}_{\mu} \times \widehat{m}=0,
$$

where $D_{\mu}$ is covariant derivative for the gauge group, $\mu=0,1,2,3$, and $g$ is magnetic charge on monopole. The vector sign and cross product in this equation are taken in internal group space and $\widehat{m}$ characterizes the additional Killing symmetry (magnetic symmetry) which commutes with the gauge symmetry itself and is normalized to unity, that is,

$$
\widehat{m}^{2}=1
$$

This magnetic symmetry obviously imposes a strong constraint on the connection and hence may be regarded as symmetry of gauge potential. This gauge symmetry restricts not only the metric but also the gauge potential. Equation (2.6) gives the following form of the generalized restricted potentials,

$$
\vec{B}_{\mu}=A_{\mu}^{*} \widehat{m}-\frac{1}{g} \widehat{m} \times \partial_{\mu} \widehat{m},
$$


with

$$
\vec{A}_{\mu}=B_{\mu}^{*} \widehat{m}
$$

where $A_{\mu}$ and $B_{\mu}$ are the electric and magnetic constituents of gauge potential. These equations give

$$
\begin{array}{r}
\widehat{m} \cdot \widehat{A}_{\mu}=B_{\mu^{\prime}}^{*} \\
\widehat{m} \cdot \widehat{B}_{\mu}=A_{\mu}^{*}
\end{array}
$$

as unrestricted Abelian components of the restricted potentials. If $\vec{A}_{\mu}=0$ in the original QCD then unrestricted potential is only $B_{\mu}^{*}$ and the restricted part of the potential is given as

$$
\vec{B}_{\mu}=-\frac{1}{g} \vec{m} \times \partial_{\mu} \widehat{m}=-\vec{W}_{\mu}
$$

where $W_{\mu}$ is the potential of topological monopoles in magnetic symmetry which is entirely fixed by $\widehat{m}$ up to Abelian gauge degrees of freedom. The unrestricted part $B_{\mu}^{*}$ of the gauge potential describes the monopole flux of color isocharges. This unrestricted part is the dual potential associated with charged gluons $W_{\mu}^{ \pm}$and leads to condensation of monopoles and the resultant state of chromo magnetic superconductivity as shown in our earlier papers [1-4].

In the presence of a complex scalar field $\phi$ (Higgs field) and in the absence of quarks or any colored object, the RCD Lagrangian in magnetic gauge may be written as

$$
L=\frac{1}{4} H_{\mu \nu} H^{\mu \nu}+\frac{1}{2}\left|D_{\mu} \phi\right|^{2}-V\left(\phi^{*} \phi\right)
$$

where

$$
\begin{gathered}
V\left(\varphi^{*} \varphi\right)=-\eta\left(|\varphi|^{2}-v^{2}\right)^{2} \\
D_{\mu} \phi=\left(\partial_{\mu}+i g W_{\mu}\right) \phi, \\
H_{\mu v}=W_{v, \mu}-W_{\mu, v},
\end{gathered}
$$

with $\eta$ as coupling constant of Higgs field and $v$ as the vacuum expectation value that is,

$$
v=\langle\phi\rangle_{0}
$$

In Prasad-Sommerfield limit [42]

$$
V(\phi)=0,
$$


but

$$
v \neq 0 .
$$

Here $W_{\mu}$ may be identified as the potential of topological monopoles in magnetic symmetry entirely fixed by $\hat{m}$ up to Abelian gauge degrees of freedom. Thus in the magnetic gauge, the topological properties of $\hat{m}$ can be brought down to the dynamical variable $W_{\mu}$ by removing all nonessential gauge degrees of freedom and hence the topological structure of the theory may be brought into dynamics explicitly where monopoles appear as point-like Abelian ones and the gauge fields are expressible in terms of purely time-like nonsingular physical potential $W_{\mu}$. Under the condition (2.19) the monopoles have lowest possible energy for given magnetic charge.

The Langarian (2.13) of RCD in the absence of quark or any colored object looks like Ginsburg-Landau Lagrangian for the theory of superconductivity. The dynamical breaking of the magnetic symmetry, due to the effective potential $V\left(\phi^{*} \phi\right)$, induces magnetic condensation of vacuum leading to the magnetic super current which screens the magnetic flux that confines the electric color iso-charges (due to dual Meissner effect). In other words, the dual Meissner effect expels the electric field between static colored charges into a narrow flux tube, giving rise to a linearly rising potential and to confinement. In this Abelian Higgs model of RCD in magnetic symmetry the $W_{\mu}$, defined by (2.16), is dual gauge field with the mass of dual gauge boson given by

$$
M_{B}=g v,
$$

and $\phi$ is the monopole field with charge $g$ and mass

$$
M_{\phi}=\sqrt{ }(8 \eta) v
$$

With these two mass scales the coherence length $\varepsilon$ and the penetration length $\lambda$ are given by

$$
\begin{gathered}
\varepsilon=\frac{1}{M_{\phi}}=\frac{1}{[\sqrt{ }(8 \eta) v]}, \\
\lambda=\frac{1}{M_{B}}=\frac{1}{(g v)} .
\end{gathered}
$$

The region in phase diagram space, where $\varepsilon=\lambda$, constitutes the border between type-I and type-II superconductors. The dual superconductivity model proposed recently by D'Alessandro et al. [21] places the Yang-Mills vacuum close to the border between type-I and type II superconductors and marginally on the type-II side. Comparing this penetration length $\lambda$ with that of relativistic superconducting model, that is,

$$
M_{s}=\sqrt{2} e|\phi|=\sqrt{2} e v=\frac{1}{\lambda_{s}},
$$


we get

$$
\frac{\lambda}{\lambda_{\mathrm{s}}}=\sqrt{2} \cot \theta
$$

where

$$
\cot \theta=\frac{e}{g^{\prime}}
$$

$e$ being the electric charge of gluons $W_{\mu}^{ \pm}$. This relation shows that with a suitable choice of the charge-space parameter $\theta$, the tube of confining flux can be made thin giving rise to a higher degree of confinement of color flux by magnetically condensed vacuum.

\section{Behaviour of Monopoles around RCD Strings in SU(2) Theory}

Lagrangian, given by (2.13), yields the following field equations:

$$
\begin{aligned}
& \partial_{\nu} H^{\mu \nu}=i \varphi^{\dagger} D_{\mu} \varphi=j_{\mu}^{0} \\
& D_{\mu}^{2} \varphi=4 \beta\left[|\varphi|^{2}-1\right] \varphi,
\end{aligned}
$$

where

$$
\begin{gathered}
j_{\mu}^{0}=i \varphi^{\dagger}\left[\partial_{\mu}+i W_{\mu}\right] \varphi, \\
\beta=\frac{\eta}{g^{2}} \\
j_{\mu}^{a}=i\left[\varphi^{\dagger} \tau^{a} D_{\mu} \varphi\right]=i\left[\phi^{\dagger} \tau^{a}\left(\partial_{\mu}+i W_{\mu}\right] \varphi\right],
\end{gathered}
$$

with $a=1,2,3$ and $\tau^{a}$, Pauli matrices, constitute the conserved Notherian current. Using relation (2.11), we may write (3.1) as

$$
\square W_{\mu}-\partial^{v} \partial_{\mu} W_{v}=i \varphi^{\dagger} \varphi\left[\frac{\partial_{\mu} \varphi}{\varphi}+i W_{\mu}\right],
$$

which reduces to the following form in the Lorentz gauge

$$
\square W_{\mu}=i \phi^{\dagger} \phi\left[\frac{\partial_{\mu} \varphi}{\varphi}+i W_{\mu}\right],
$$

which further reduces into following simple form for the small variation in $\phi$

$$
\square W_{\mu}+|\varphi|^{2} W_{\mu}=0,
$$


which is a massive vector-type equation where the equivalent mass of the vector particle state (i.e., condensed mode) may be identified as

$$
M=|\phi|
$$

with its expectation value

$$
\langle M\rangle=v,
$$

which gives

$$
M_{B}=g\langle M\rangle=v g=\frac{1}{\lambda^{\prime}}
$$

where $\lambda$ is penetration length. Thus the penetration length directly follows from the field equation (3.1) obtained from the Lagrangian (2.5) of the extended Abelian Higgs model in restricted chromodynamics.

Magnetically condensed vacuum of action of Lagrangian of (2.13) is characterized by the presence of two massive modes. The mass of scalar mode, $M_{\phi}$ given by (2.21), determines how fast the perturbative vacuum around a colored source reaches the condensation and the mass $M_{D}$ of the vector mode determines the penetration length of the colored flux. The masses of these magnetic glue balls may be estimated [33, 36, 37] by evaluating string tension of the classical string solutions of quark pairs, since the extended Abelian Higgs model in restricted chromo dynamics, admits string-like solutions [38]. Let us examine the behavior of monopoles around such RCD strings. The classical field equations (3.1) and (3.2) contain a solution corresponding to the RCD string with a quark and an antiquark at its ends. We consider such strings which are stationary and translationaly invariant along the third direction $Z=x^{3}$ of the reference frame used in Lagrangian (2.13). Let us consider the following ansatz $[39,43]$ for the four components of the vector field $\widehat{W}_{\mu}$ and the two complex components $\phi_{1}$ and $\phi_{2}$ of the Higgs field $\phi$;

$$
\begin{gathered}
W_{\mu}=\left\{W_{i}(\rho), W_{\alpha}(\rho)\right\}, \\
\phi_{i}=f_{i}(\rho)\left[e^{i\left(w_{\alpha} x_{\alpha}\right) \delta_{i 2}}\right] e^{i \psi(p)},
\end{gathered}
$$

where $i=1,2, \alpha=3,4, f_{i}(\rho)$ are complex functions of $\rho=\left(x_{1}^{2}+x_{2}^{2}\right)^{1 / 2}$ and $\omega_{3}$ and $\omega_{4}$ are real parameters. Here $\omega_{4}$ is the relative rotation and $\omega_{3}$ is the relative twist along $z$-axis between the components $\phi_{1}$ and $\phi_{2}$ of the Higg's field $\phi$. This ansatz breaks the originally present global SU(2) symmetry to $U(1)$ and the various terms of the Lagrangian (2.13) reduce 
from four-dimensional configuration to the two-dimensional configuration in the following manner;

$$
\begin{gathered}
H_{\mu \nu} H^{\mu \nu} \longrightarrow H_{i j}^{2}-2\left[\left(\partial_{i} W_{\alpha}\right)\left(\partial_{i} W^{\alpha}\right)\right] \\
\left(D_{\mu} \varphi\right)\left(D^{\mu} \varphi\right) \longrightarrow \omega^{\alpha}\left(\omega_{\alpha}+2 W_{\alpha}\right)\left|\varphi_{2}\right|^{2}-\left|D_{i} \varphi_{a}\right|^{2}+W_{\alpha} W^{\alpha}\left|\varphi_{a}\right|^{2}, \\
{\left[|\phi|^{2}-1\right]^{2} \longrightarrow\left[\left|\phi_{a}\right|^{2}-1\right]^{2}}
\end{gathered}
$$

where $i, j$, and $a=1,2, W^{\alpha} W_{\alpha}=W_{3}^{2}-W_{4}^{2}$, and $\omega^{\alpha} \omega_{\alpha}=\omega_{3}^{2}-\omega_{4}^{2}$.

Then the action of Lagrangian (2.13) reduces to

$$
\begin{aligned}
A \longrightarrow \frac{v^{2}}{|q|^{2}} \int d x^{4} d x^{3} \int d^{2} x[ & W_{\alpha} W^{\alpha}\left|\varphi_{a}\right|^{2}+\frac{1}{2} \omega^{\alpha}\left(\omega^{\alpha}+2 W_{\alpha}\right)|\varphi|^{2} \\
& \left.-\frac{1}{2}\left|D_{i} \varphi_{a}\right|^{2}+\frac{1}{4} H_{i j}^{2}-\frac{1}{2}\left(\partial_{i} W_{\alpha}\right)\left(\partial_{i} W^{\alpha}\right)+\beta\left(\left|\varphi_{a}\right|^{2}-1\right)^{2}\right],
\end{aligned}
$$

where

$$
H_{12}=-H_{21}=\frac{\partial W_{2}}{\partial x_{1}}-\frac{\partial W_{1}}{\partial x_{2}}
$$

With this Ansatz the field equations (3.1) and (3.2) take the following forms in the $x_{1}-x_{2}$ plane

$$
\begin{gathered}
\square W_{\alpha}=-\omega_{\alpha}\left|\varphi_{2}\right|^{2}-W_{\alpha}\left|\varphi_{\alpha}\right|^{2}, \\
\partial_{j} H_{j k}=i\left[\phi_{a} D_{k} \phi_{a}\right], \\
D_{i}^{2} \varphi_{1}=-4 \beta\left[\left|\varphi_{a}\right|^{2}-1\right] \varphi_{1}-2 W_{\alpha} W^{\alpha} \varphi_{1}, \\
D_{2}^{2} \varphi_{2}=-4 \beta\left[\left|\varphi_{a}^{2}\right|-1\right] \varphi_{2}-2 W_{\alpha} W^{\alpha} \varphi_{2}+\omega^{\alpha}\left(\omega_{\alpha}+2 W_{\alpha}\right) \varphi_{2},
\end{gathered}
$$

where (3.16) may also be written as

$$
\Delta W_{\alpha}=\omega_{\alpha}\left|\varphi_{2}\right|^{2}+W_{\alpha}\left|\varphi_{a}\right|^{2}
$$

with $\Delta=-\square$.

Let us consider the solutions of these equations in the following simple case of the ansatz used in (3.10) and (3.11)

$$
W_{1}=\frac{\widehat{x}_{2} h(\rho)}{|g| \rho^{2}}, \quad W_{2}=-\frac{\widehat{x}_{1} h(\rho)}{|g| \rho^{2}}, \quad W_{3}=0, \quad W_{4}=0
$$


where $\widehat{x}_{1}$ and $\widehat{x}_{2}$ are unit vectors along $x_{1}$ and $x_{2}$ directions. In this case (3.20) gives

$$
\omega_{\alpha}=0,
$$

and then (3.18) and (3.19) reduce to

$$
D_{i}^{2} \varphi_{a}=-4 \beta\left[\left|\varphi_{a}\right|^{2}-1\right] \varphi_{a}
$$

and relation (3.11) becomes

$$
\varphi_{i}=f_{i}(\rho) e^{i \psi(\rho)}
$$

showing that there is neither relative rotation nor relative twist between the components of $\phi_{1}$ and $\phi_{2}$ of the Higgs field $\phi$. The solutions (3.21) and (3.24) are static and untwisted semilocal solutions. Here $\rho$ is the transverse distance to the string and

$$
\psi=\arg \left(x_{1}+i x_{2}\right)
$$

$$
\begin{aligned}
& \lim _{\rho \rightarrow 0} f(\rho)=\lim _{\rho \rightarrow 0} h(\rho)=0, \\
& \lim _{\rho \rightarrow \alpha} f(\rho)=\lim _{\rho \rightarrow 0} h(\rho)=1,
\end{aligned}
$$

where

$$
f(\rho)=f_{1}(\rho), \quad h(\rho)=f_{2}(\rho) .
$$

From (3.1) we get the monopole current as

$$
k_{\mu}=g \operatorname{Im}\left[\phi^{+} D_{\mu} \phi\right]=g|\phi|^{2}\left[\partial_{\mu} \arg \phi+g W_{\mu}\right] .
$$

Equation (3.25) gives

$$
\frac{\partial \psi}{\partial x_{1}}=-\frac{x_{1}}{\rho^{2}}, \quad \frac{\partial \psi}{\partial x_{2}}=-\frac{x_{2}}{\rho^{2}}
$$

Substituting relations (3.24), (3.21), (3.25), (3.26b), and (3.28) into (3.27), we get

$$
\begin{gathered}
k_{i}=-\frac{\left(v^{2} \epsilon_{i j} x_{j}\right)}{\rho^{2}} g f^{2}(\rho)[1-h(\rho)], \\
k_{2}=0, \quad k_{4}=0,
\end{gathered}
$$


where $\epsilon_{12}=-\epsilon_{21}=1$ and $\epsilon_{11}=\epsilon_{22}=0$, summation over repeated index is conventionally involved. Substituting relations (3.24), (3.21) and (3.28) into field equation (3.23), we have

$$
f^{\prime \prime}(\rho)+\frac{f^{\prime}(\rho)}{\rho}-\frac{f(\rho)}{\rho^{2}[1-h(\rho)]^{2}}+\left(\frac{M_{\phi}^{2}}{2}\right)\left[1-f^{2}(\rho)\right] f(\rho)=0,
$$

where dash devotes derivatives with respect to $\rho$. At large distance, in view of equations (3.20), we may have

$$
f(\rho)=1-\varepsilon(\rho)
$$

where $\varepsilon(\rho)$ is infinitesimally small at large distance such that

$$
\lim _{\rho \rightarrow \infty} \varepsilon(\rho)=0
$$

Then (3.30) may be written as

$$
\varepsilon^{\prime \prime}(\rho)+\frac{\varepsilon^{\prime}(\rho)}{\rho}-M_{\phi}^{2} \varepsilon(\rho)=0
$$

Substituting $r=M_{\phi} \rho$ into this equation, we have

$$
\frac{d^{2} \varepsilon(r)}{d r^{2}}+\left(\frac{1}{r}\right) \frac{d \varepsilon(r)}{d r}-\varepsilon(r)=0
$$

which is modified Bessel's equation of zero order, with its solution given as

$$
\varepsilon(r)=A I_{0}(r)=A I_{0}\left(M_{\phi} \rho\right)
$$

where $I_{0}$ is the modified Bessel's function of zero order. In the similar manner, the field equation (3.4) may be written into the following form by using relations (3.21) and (3.29);

$$
h^{\prime \prime}(\rho)-\frac{h^{\prime}(\rho)}{\rho}+M_{B}^{2}[1-h(\rho)] f^{2}(\rho)=0 .
$$

At large distance we may have

$$
h(\rho)=1-\xi(\rho)
$$

where $\lim _{\rho \rightarrow \infty} \xi(\rho)=0$. 
Then (3.36) reduces to

$$
\frac{d^{2} \xi(r)}{d r^{2}}-\frac{d \xi(r)}{d r}-\xi(r)=0
$$

where $r=M_{B} \rho$. Let us substitute $\xi(r)=r_{X}(r)$ into this equation. Then we have

$$
r \frac{d^{2} \chi(r)}{d r^{2}}+\frac{d \chi(r)}{d r}-\chi(r)\left[1+\frac{1}{r^{2}}\right]=0
$$

which is modified Bessel's equation of order-one with its solution given by

$$
\chi(r)=\frac{\xi(r)}{r}=B I_{1}(r)
$$

where $I_{1}(r)$ is modified Bessel's function of order one. Thus we have

$$
\xi(\rho)=B\left(M_{B} \rho\right) I_{1}\left(M_{B} \rho\right) .
$$

Substituting relations (3.35) and (3.41) into (3.31) and (3.37), we have at large value of $\rho$,

$$
\begin{gathered}
f(\rho)=1-A I_{0}\left(M_{\phi} \rho\right), \\
h(\rho)=1-B\left(M_{B} \rho\right) I_{1}\left(M_{B} \rho\right) .
\end{gathered}
$$

Substituting these results into (3.21) and (3.24) with (3.25), we get the solution of classical field equations (3.2) and (3.23) corresponding to the RCD string with a quark and an antiquark at its ends. The infinitely separated quark and antiquark correspond to an axially symmetric solution of the string. For such a string solution with a lowest nontrivial flux, the coefficient $A$ in the solution (3.35) is always equal to one while the coefficient $B$ in the solution (3.41) is unity in the BogomoLny limit exactly on the border between the type I and type II superconductors where $M_{B}=M_{\phi}$, that is, coherence length and the penetration length coincide with each other. Thus in RCD close to border, we set $B=1$ besides $A=1$ and then we have

$$
\begin{gathered}
f(\rho)=1-I_{0}\left(M_{\phi} \rho\right)=-\sum_{n=1}^{\infty} \frac{\left(M_{\phi} \rho / 2\right)^{2 n}}{(n !)^{2}} \\
h(\rho)=1-\left(M_{B} \rho\right) I_{1}\left(M_{B} \rho\right)=1-\frac{\left(M_{B} \rho / 2\right)^{2}}{2}-M_{B} \rho \sum_{n=1}^{\infty} \frac{\left(M_{B} \rho / 2\right)^{2 n+1}}{\Gamma(n) \Gamma(n+1)} .
\end{gathered}
$$

The RCD string is well defined by these solutions. 


\section{Monopole Density around RCD String}

Substituting these relations (3.44) into (3.29), we can find the monopole density in the vicinity of RCD string. To meet this end, let us write the partition function corresponding to RCD Lagrangian of (2.13) in Abelian Higgs Model (AHM) in the following form by using (2.15);

$$
\begin{aligned}
& Z=\int D W_{\mu} D \phi \exp \left\{-\int d^{4} x L_{\mathrm{AHM}}\left(W_{\mu}, \phi\right)\right\} \text { with } \\
& L_{\mathrm{AHM}}\left(W_{\mu}, \phi\right)=\frac{1}{4} H_{\mu \nu} H^{\mu v}+\frac{1}{2}\left|\left(\partial_{\mu}+i g W_{\mu}\right) \phi\right|^{2}+\eta\left(|\phi|^{2}-v^{2}\right)^{2} .
\end{aligned}
$$

This model (AHM) incorporates dual superconductivity and hence confinement as the consequence of monopole condensation since the Higgs-type mechanism arises here.

With this partition function the quantum average of Wilson loop may be written as

$$
\left\langle W_{l}^{c}\right\rangle=\left\langle K_{\left(q_{l}, q_{m}\right)}^{c}\left(W_{\mu}\right)\right\rangle_{\mathrm{AHM}^{\prime}}
$$

where the expectation value in r.h.s is calculated in AHM with the operator $K_{\left(q_{e}, q_{m}\right)}^{c}$ as the product of 't Hooft loop and the Wilson loop $W^{c}$;

$$
K_{\left(q_{e}, q_{m}\right)}^{c}\left(W_{\mu}\right)=H_{q_{e}}^{c}\left(W_{\mu}\right) \cdot W_{q_{m}}^{c}\left(W_{\mu}\right), \quad \text { where } q_{e}=e_{0} ; q_{m}=\frac{e_{0}}{g} e,
$$

and Wilson loop given as

$$
W_{l}^{c}\left(W_{\mu}\right)=\exp \left\{i e_{0} \int d^{4} x \eta_{\mu} W^{\mu}\right\} \quad \text { with } \eta(x)=\oint_{C} d \breve{x}_{\mu} \delta^{(4)}(x-\breve{x}(\tau)),
$$

which creates the particle with electric charge $e_{0}$ on the world trajectory $C$.

Then the effective electric and magnetic four-current density may be written as follows;

$$
j_{\mu}=q_{e} \eta_{\mu} ; \quad k_{\mu}=q_{m} \eta_{\mu} .
$$

In (4.3) the operator $H_{q_{e}}^{c}\left(W_{\mu}\right)$ is

$$
H_{q_{e}}^{c}\left(W_{\mu}\right)=\exp \left\{-\frac{1}{4} \int d^{4} x\left[\left(H_{\mu \nu}-\frac{1}{2} \varepsilon_{\mu \nu \alpha \beta} F_{\alpha \beta}\right)^{2}-H_{\mu \nu} H^{\mu \nu}\right]\right\}
$$

and $F_{\alpha \beta}$ is the dual field tensor satisfying

$$
F_{\mu v, v}=j_{\mu} .
$$

This operator $H_{q_{e}}^{c}$ creates the string spanned by the loop $C$, carrying the flux $q_{e}$. In AHM the monopoles are condensed and in its string representation the topological interaction exists in 
the expectation value of the Wilson loop $W^{c}$ given by (4.4). In the centre of ANO string the Higgs field $\phi=|\phi| e^{i \theta}$ vanishes, that is,

$$
\operatorname{Re} \phi=\operatorname{Im} \phi=0,
$$

and the phase is singular on the world sheets of ANO string. Then the measure of the integration over $\phi$ can be written as

$$
D \phi=C D|\phi|^{2} D \theta
$$

where $C$ is a constant. The integral $\int D \theta$ contains the integration over functions which are singular on two dimensional manifolds. Let us divide the phase into regular and singular parts as

$$
\theta=\theta^{r}+\theta^{s}
$$

where $\theta^{s}$ is defined by

$$
\left(\partial_{\mu} \partial_{v}-\partial_{\nu} \partial_{\nu}\right) \theta^{s}(x, \breve{x})=2 \pi \varepsilon_{\mu \nu \alpha \beta} \sum_{\alpha \beta}(x, \breve{x}),
$$

where

$$
\sum_{\alpha \beta}(x, \breve{x})=\int_{\Sigma} d^{2} \sigma \varepsilon^{a b} \partial_{a} \breve{x}_{\alpha} \partial_{b} \breve{x}_{\beta} \delta^{(4)}[x-\breve{x}(\sigma)]
$$

with $\partial_{a}=\partial / \partial \sigma^{a}, \breve{x}_{\mu}$ string-position and $\sum$ as the collection of all the closed surfaces.

$\sigma=\left(\sigma_{1}, \sigma_{2}\right)$ is the parameterization of string surface and $a, b=1,2$. Then the measure $D \theta$ can be decomposed as

$$
D \theta=D \theta^{r} D \theta^{s}
$$

Let us use these relations to find the monopole density in the vicinity of RCD string for vanishing and nonvanishing coherence lengths respectively in the following subsections.

\subsection{For Zero Coherence Length}

From (2.21), we find the vanishing coherence length in the limit $M_{\phi} \rightarrow \infty$ or $\eta \rightarrow \infty$ which corresponds to infinitely deep potential $V\left(\phi^{*} \phi\right)$ of (2.14). This limit is London limit. Then the RCD Lagrangian of (4.1) in AHM may be written as follows

$$
L_{m}=\frac{1}{4} H_{\mu \nu} H^{\mu \nu}+\frac{v^{2}}{2}\left(\partial_{\mu} \varphi+g W_{\mu}\right)^{2}=L_{m}\left(W_{\mu}, \varphi\right)
$$


where $L_{m}$ denotes the Lagrangian density for monopoles. In terms of this Lagrangian, the partition function of (4.1) may be written as follows

$$
Z=\int_{-\pi}^{\pi} D \phi\left[\int_{-\infty}^{\infty} D W_{\mu} \exp \left\{-d^{4} x L_{m}\left(W_{\mu}, \varphi\right)\right\}\right] .
$$

The string in RCD manifests itself as a singularity in the phase of the Higgs field according to (4.11).

Let us fix the unitary gauge as

$$
\phi=0
$$

and make the consequent shift

$$
W_{\mu} \longrightarrow W_{\mu}-\frac{1}{g} \partial_{\mu} \phi
$$

Then the shift in $H_{\mu \nu}$ will be

$$
H_{\mu \nu} \longrightarrow H_{\mu v}-\frac{2 \pi}{g} \sum_{\mu \nu}^{(d)}
$$

where

$$
\sum_{\mu \nu}^{(d)}(x)=\frac{1}{2} \epsilon_{\mu \nu \rho \sigma} \sum_{\rho \sigma}(x)
$$

and we have used relations (4.11), (4.16), and (4.13). Substituting shifts (4.16) and (4.17) into (4.14) and integrating over the field $W_{\mu}$, we get

$$
Z=\int_{\partial \Sigma=0} D \Sigma \exp \left\{-A_{\text {str }}(\Sigma)\right\}
$$

where $A_{\text {str }}$ is the string action given as

$$
A_{\mathrm{str}}=2 \pi^{2} v^{2} \int d^{4} x \int d^{4} y \Sigma_{\mu v}(x) D_{M_{B}}(x-y) \Sigma_{\mu v}(y)
$$


where $D_{M_{B}}(x)$ is the scalar Yukawa propagator. It is the propagator of the scalar particle of mass $M_{B}=g v$, that is,

$$
\left(\Delta+m^{2}\right) D_{M_{S}}(x)=\delta^{4}(x)
$$

with $m=g v$ as mass of dual gauge boson $W_{\mu}$.

For closed strings, we have

$$
\partial_{v} \Sigma_{\mu v}=0 .
$$

On the other hand, when the strings are spanned on the current $j_{c}$, we have

$$
\partial_{\nu} \Sigma_{\mu \nu}=j_{\mu}^{c}
$$

The action of the currents is given as follows by the short-ranged exchange of the dual gauge boson,

$$
A_{\text {curr }}\left(j^{c}\right)=\frac{e^{2}}{2} \int d^{4} x \int d^{4} y j_{\mu}^{(c)}(x) D_{M_{B}}(x-y) j_{y}^{c}(y)
$$

where $e$ is the electric charge of gluon, satisfying the quantization condition

$$
e g=2 \pi
$$

The quantum average of Wilson loop can be written here as a sum over strings similar to (4.19),

$$
\left\langle W_{l}^{c}\right\rangle=\frac{1}{Z} \int_{\partial \Sigma=j c} D \Sigma \exp \left\{-A_{\text {str }}(\Sigma)-A_{\text {curr }}(j c)\right\}
$$

where $Z$ is given by (4.19). In this equation the sources of electric flux (i.e., quarks) running along the trajectory $C$ are introduced with the help of $W_{l}^{c}$.

Let us place the static quarks at spatial infinities of the axis- $x_{3}$. Then the effects of quarks (i.e., boundary effects) are avoided and consequently the second term of the exponential in r.h.s of (4.26) may be ignored. In this case (i.e., infinite static string placed along the third direction) (4.11) reduces to the following form of string current

$$
\Sigma_{\mu v}=\left(\partial_{\mu, 3} \partial_{v, 4}-\partial_{\mu, 4} \partial_{v, 3}\right] \delta\left(x_{1}\right) \delta\left(x_{2}\right)=\delta_{3,4}^{\mu, v} \delta\left(x_{1}\right) \delta\left(x_{2}\right) .
$$

From (3.27), the monopole current may be written as follows in the London limit;

$$
k_{\mu}=g v^{2}\left[\partial_{\mu} \varphi+g W_{\mu}\right]
$$


When the singular phase $\phi_{\Sigma}$ corresponds to the string position fixed by (4.11), the Lagrangian $L_{m}$ in the exponential of (4.14) becomes

$$
L_{m}\left(W_{\mu}, \varphi\right)=L_{m}\left(W_{\mu}, \varphi_{\Sigma}\right)
$$

and then the functional generating the partition function in (4.14) may be written as

$$
Z[\Sigma, C]=\int_{-\infty}^{\infty} D W_{\mu} \exp \left\{-\int d^{4} x\left[L_{m}\left(W_{\mu}, \varphi_{\Sigma}\right)-i k_{\mu} C_{\mu}\right]\right\}
$$

Then the monopole current in the presence of the string is given by the variational derivative [44]

$$
\left\langle k_{\mu}(x)\right\rangle_{\Sigma}=\left.\frac{g}{Z(\Sigma, 0)}\left(\frac{\delta}{i \partial C_{\mu}(x)}\right)^{2} Z[\Sigma, C]\right|_{C=0}
$$

And the squared monopole density is

$$
\left\langle k_{\mu}^{2}(x)\right\rangle_{\Sigma}=\left.\frac{g}{Z(\Sigma, 0)}\left(\frac{\delta}{i \partial C_{\mu}(x)}\right)^{2} Z[\Sigma, C]\right|_{C=0} .
$$

In the manner analogous to (4.19) and (4.20), the generating functional (4.30) may be written as

$$
\begin{array}{r}
Z[\Sigma, C]=\exp \left[-\int d^{4} x \int d^{4} y\left\{\frac{g^{2} v^{4}}{2} C_{\mu}(x) D_{M_{B}}^{\mu v}(x-y) C_{v}(y)\right\}\right. \\
\left.-2 \pi i v^{2} C_{\mu}(x) D_{M_{s}}^{\mu v}(x-y) \partial_{\rho} \sum_{\rho v}^{(d)}(y)-A_{\mathrm{str}}(\Sigma)\right]
\end{array}
$$

where string action $A_{\text {str }}(\Sigma)$ is given by (4.20).

Substituting this relation for generating functional into (4.32) and evaluating the monopole density, we get the monopole current around the string as

$$
k_{\mathrm{str}}^{\mu}=\left\langle k^{\mu}\right\rangle_{\Sigma}=-2 \pi g v^{2} \int d^{4} y D_{M_{B}}^{\mu v}(x-y) \partial_{\rho} \Sigma_{\rho v}^{d}(y)
$$


For static string, this equation reduces to

$$
\begin{gathered}
k_{\mathrm{str}}^{i}=-2 \pi g v^{2} \in^{i j} \frac{x_{i}}{\rho} \frac{\partial}{\partial \rho} D_{M_{B}}(\rho), \quad i, j=1,2, \\
k_{\mathrm{str}}^{3}=0, \quad k_{\mathrm{str}}^{4}=0,
\end{gathered}
$$

where

$$
D_{M_{B}}(\rho)=\frac{1}{2 \pi} I_{0}\left(M_{B} \rho\right),
$$

with $I_{0}$ as modified Bessel's function of zero order. The function $D_{M_{B}}(\rho)$ given by (3.54), is the propagator for a scalar massive particle in two space-time dimensions. Using (4.34) and (4.36), the explicit form of the nonzero component of the solenoidal current may be written as

$$
k_{\theta}^{\mathrm{str}}=v^{2} g M_{B} I_{1}\left(M_{B} \rho\right)
$$

where $I_{1}\left(M_{B} \rho\right)$ is the modified Bessel's function of order one given in (3.44). Thus the monopoles form a solenoidal current which circulates around the string in transverse directions. This current gives rise to the following squared monopole density;

$$
\left\langle k^{2}\right\rangle_{\Sigma}=v^{4} g^{2} M_{B}^{2} I_{1}^{2}\left(M_{B} \rho\right)
$$

Substituting the value of $I_{1}\left(M_{B} \rho\right)$ from (3.44) into this relation, we find that the squared density of the monopole current, in London limit (where coherence length is zero), has a maximum at the distance of the order of the $1 / M_{B}$ (i.e., the order of penetration length).

\subsection{For Nonzero Coherence Length}

When the potential $V\left(\phi^{*} \phi\right)$ of (2.14) is of finite depth, that is, $\eta$ is finite then $M_{\phi}$ is finite and hence coherence length $\xi$ given by (2.23), is nonzero and finite. Then in the expression (4.38) for squared monopole density in the vicinity of RCD string a term corresponding to quantum vacuum correction is nonzero even in the absence of string. Thus the squared monopole density, in this case, is written as

$$
\left(k_{\mu}^{2}\right)_{\Sigma}=\left(k_{\mu}^{\text {string }}\right)^{2}+\left(k_{\mu}^{\text {quan }}\right)^{2}
$$


where $\left(k_{\mu}^{\text {string }}\right)^{2}$ is given by (4.38) and quantum vacuum correction $\left(k_{\mu}^{\text {quan }}\right)^{2}$ is given by

$$
\left(k_{\mu}^{\text {quan }}\right)^{2}=\left\langle k_{\mu}^{2}\right\rangle_{0}=g^{2} v^{4} D_{M_{B}}^{2}(0)=\frac{g^{2} v^{4} \wedge^{2}}{16 \pi^{2}}
$$

where we have used (4.36) and regularized the divergent expression by momentum cut off $\wedge$.

Replacing vacuum expectation value $v$ of the Higgs field $\phi$ by $|\phi(\rho)|$ in relation (4.40) and then substituting it into (4.39), we get

$$
\begin{aligned}
\left(k_{\mu}^{2}\right)_{\Sigma} & =\left(k_{\mu}^{\text {string }}\right)^{2}+g^{2} \frac{|\varphi(\rho)|^{4} \wedge^{2}}{16 \pi^{2}} \\
& =g^{2} \nu^{4} M_{B}^{2} I_{1}^{2}\left(M_{B} \rho\right)+g^{2} \frac{|\varphi(\rho)|^{4} \wedge^{2}}{16 \pi^{2}} .
\end{aligned}
$$

For $\rho$ of the order of coherence length $\xi$ the quantum correction to the squared monopole density is much more than the vacuum expectation value measured far out side the string $(\rho \gg \Sigma)$. Thus the quantum corrections control the leading behavior of the total monopole density in the vicinity of the RCD string.

Using the asymptotic expansions of modified Bessel's functions in (3.44), and then introducing it into (3.24), we get

$$
|\phi|^{4} \approx\left[1-4 \frac{\sqrt{\pi \xi}}{2 \rho} e^{-\rho / \xi}\right]
$$

Then (4.42) may be approximately written as follows [38] at large distances;

$$
\left(k_{\mu}^{2}\right)_{\Sigma} \approx \frac{g^{2} \wedge^{2}}{16 \pi^{2}}\left[1-4 \frac{\sqrt{\pi \xi}}{2 \rho} e^{-\rho / \xi}\right]
$$

which shows that the leading behaviors of the monopole density at large distances are controlled by coherence length $\xi$ and not by penetration length $\lambda$.

\section{Discussion}

The Lagrangian, given by (2.13) for RCD in magnetic gauge in the absence of quarks or any colored objects, establishes an analogy between superconductivity and the dynamical breaking of magnetic symmetry which incorporates the confinement phase in RCD vacuum where the effective potential $V\left(\theta^{*} \theta\right)$, given by (2.14), induces the magnetic condensation of vacuum. This gives rise to magnetic super current which screens the magnetic flux and confines the color iso-charges as the result of dual Meissner effect. The confinement of color is due to the spontaneous breaking of magnetic symmetry which yields a nonvanishing magnetically charged Higg's condensate, where the broken magnetic group is chosen by Abelianization process and hence the magnetic condensation mechanism of confinement in RCD is dominated by Abelian degrees of freedom. Such Abelian dominance in connection 
with monopole condensation has recently been demonstrated by Bornyakov et al. [45]. The similar result has also been obtained more recently in a dual superconductivity model [21].

In the confinement phase of RCD, the monopoles are condensed under the condition (2.19) where the transition from $\langle\phi\rangle_{0}=0$ to $\langle\phi\rangle_{0}=v \neq 0$ is of first order, which leads to the vacuum becoming a chromo magnetic superconductor in the analogy with Higg's-GinsburgLandau theory of superconductivity. Magnetically condensed vacuum is characterized by the presence of two massive modes given by (2.20) and (2.21), respectively, where the mass of scalar mode $M_{\phi}$ determines how fast the perturbative vacuum around a color source reaches condensation and the mass $M_{B}$ of vector mode determines the penetration length of the colored flux. With these two scales of dual gauge boson and monopole field, the coherence length $\varepsilon$ and the penetration length $\lambda$ have been constructed by (2.23) in RCD theory. These two lengths coincide at the border between type-I and type-II-superconductors.

The ansatz given by relations (3.10) and (3.11) shows that there is a nontrivial coordinate dependent relative phase between the components of $\mathrm{SU}(2)$ doublets. This anastz breaks the originally present global SU(2) symmetry to $U(1)$ and reduces the fourdimensional action of Lagrangian (2.13) to the two dimensional one given by (3.14) with the field equation given by (3.16), (3.17), (3.18), and (3.19). For the special case with the static solution given by (3.21), (3.23) and (3.24) there is neither a relative rotation nor a relative twist between the components of Higg's field. Relations (3.35) and (3.41) remove the mistakes of the similar relations of Chernodub et al. [44]. Substituting relations (3.44) into (3.21) and (3.24), the solutions of classical field equations (3.1) and (3.2), corresponding to the RCD string with a quark and antiquark at its ends, readily follows. The RCD string is well defined by solutions (3.44) where the monopole current given by (3.27) near the RCD string, is zero at the center of the string and also zero at points far away from the string.

Equation (4.14) gives the partition function in the Eulidean space-time with the RCD Lagrangian density in magnetic gauge given by (4.13). This partition function has been computed in the form given by (4.19) in terms of string action given by (4.20). The action of the current around the strings is given by (4.24) which leads to the quantum average of Wilson loop as given by (4.26) where the source of electric flux (i.e., quarks) running along the trajectory are introduced with the help of Wilson loop.

Equation (4.28) gives the monopole current in London limit which corresponds to infinitely deep Higg's potential and leads to vanishing coherence length in the chromo magnetic superconductor. Equation (4.31) gives the monopole current in the presence of the string, which leads to squared monopole density given by (4.32). The monopole current given by (4.34) reduces to the components given by (4.35) in terms of propagator (4.36) for a scalar massive particle in two space-time dimensions. Equation (4.37) gives the explicit form of the nonzero component of the solenoidal current which circulates around the string in transverse directions. This current gives rise to the squared monopole current given by (4.38) in London limit (i.e., vanishing coherence length). This squared current has a maximum at the distance of the order of penetration length. Thus in London limit (zero coherence length) the monopole density around the string in RCD is governed by penetration length.

Equation (4.39) shows that for nonzero finite coherence length, the monopole density is nonzero even in the absence of string. Equation (4.42) shows that the quantum correction to the squared monopole density is much more than the vacuum expectation value measured far out side the string. Thus the quantum corrections control the leading behavior of the total monopole density in the vicinity of the RCD string. Equation (4.43) shows that the leading behavior of the monopole density at large distances is controlled by the coherence length 
and not by the penetration length. This result is in agreement with the numerical result of Bornyakov et al. [45, 46].

\section{References}

[1] B. S. Rajput, "Relationship between restricted chromodynamics and the dual superconductivity," Indian Journal of Pure and Applied Physics, vol. 34, no. 8, pp. 528-533, 1996.

[2] B. S. Rajput, "Superconductivity at Higher temperature as restoration of supersymmetry," Indian Journal of Pure and Applied Physics, vol. 33, pp. 166-168, 1995.

[3] S. Kumar, R. Swarup, B. S. Rajput, and B. Singh, "Dual superconductivity in restricted chromodynamics (RCD)," International Journal of Theoretical Physics, vol. 48, no. 6, pp. 1766-1780, 2009.

[4] S. Kumar, "Superconductivity in restricted chromo-dynamics (RCD) in SU(2) and SU(3) gauge theories," International Journal of Theoretical Physics, vol. 49, no. 3, pp. 512-525, 2010.

[5] Y. Nambu, "Strings, monopoles, and gauge fields," Physical Review D, vol. 10, no. 12, pp. 4262-4268, 1974.

[6] Y. Nambu, "Hydrodynamic boundary conditions and mode-mode coupling theory," Physics Reports, vol. 23, no. 3, pp. 250-253, 1976.

[7] G. 't Hooft, "On the phase transition towards permanent quark confinement," Nuclear Physics B, vol. 138, no. 1, pp. 1-25, 1978.

[8] B. S. Rajput, J. M. S. Rana, and H. C. Chandola, "Quark confinement through dyonic condensation," Progress of Theoretical Physics, vol. 82, no. 1, p. 153, 1989.

[9] B. S. Rajput, J. M. S. Rana, and H. C. Chandola, "The Quark confinement in extended gauge theory," Canadian Journal of Physics, vol. 69, p. 1441, 1991.

[10] G. 't. Hooft, "Magnetic charge quantization and fractionally charged quarks," Nuclear Physics B, vol. 105 , no. 3, pp. 538-547, 1976.

[11] G. 't. Hooft, "Topology of the gauge condition and new confinement phases in non-abelian gauge theories," Nuclear Physics B, vol. 190, no. 3, pp. 455-478, 1981.

[12] S. Mandelstam, "II. Vortices and quark confinement in non-Abelian gauge theories," Physics Reports, vol. 23, no. 3, pp. 245-249, 1976.

[13] S. Mandelstam, "Charge monopole duality and the phases of non-Abelian Gauge theories," Physical Review D, vol. 19, no. 8, pp. 2391-2409, 1979.

[14] S. Mandelstam, "Approximation scheme for quantum chromodynamics," Physical Review C, vol. 35, p. 269, 1984.

[15] Z. F. Ezawa and A. Iwazaki, "ZN topology and charge confinement in SU(N) Higgs models," Physical Review D, vol. 23, no. 12, pp. 3036-3045, 1981.

[16] Z. F. Ezawa and A. Iwazaki, "ZN topology and charge confinement in SU(N) Higgs models. II," Physical Review D, vol. 24, no. 8, pp. 2264-2273, 1981.

[17] Z. F. Ezawa and A. Iwazaki, "Abelian dominance and quark confinement in Yang-Mills theories," Physical Review D, vol. 25, no. 10, pp. 2681-2689, 1982.

[18] Z. F. Ezawa and A. Iwazaki, "Abelian dominance and quark confinement in Yang-Mills theories. II. Oblique confinement and $\eta^{\prime}$ mass," Physical Review D, vol. 26, no. 3, pp. 631-647, 1982.

[19] V. P. Nair and C. Rosenzweig, "QCD vacuum as a chromomagnetic superconductor: microscopic physics," Physical Review D, vol. 31, no. 2, pp. 401-410, 1985.

[20] Y. Simonov, "The confinement," Physics-Uspekhi, vol. 39, no. 4, p. 313, 1996.

[21] A. D'Alessandro, M. D'Elia, and L. Tagliacozzo, "Dual superconductivity and vacuum properties in Yang-Mills theories," Nuclear Physics B, vol. 774, no. 1-3, pp. 168-181, 2007.

[22] P. YU. Boyko, V. G. Bornyakov, E. M. Ilgenfritz et al., "Once more on the interrelation between Abelian monopoles and P-vortices in SU (2) LGT," Nuclear Physics B, vol. 756, no. 1-2, pp. 71-85, 2006.

[23] G. S. Bali, V. Bornyakov, M. Müller-Preussker, and K. Schilling, "Dual superconductor scenario of confinement: a systematic study of Gribov copy effects," Physical Review D, vol. 54, no. 4, pp. 28632875, 1996.

[24] T. Suzuki and I. Yotsuyanagi, "Possible evidence for Abelian dominance in quark confinement," Physical Review D, vol. 42, no. 12, pp. 4257-4260, 1990.

[25] T. Suzuki, "Monopoles and confinement," Nuclear Physics B, vol. 30, no. C, pp. 176-183, 1993.

[26] Y. Koma, M. Koma, E. M. Ilgenfritz, and T. Suzuki, “Detailed study of the Abelian-projected SU(2) flux tube and its dual Ginzburg-Landau analysis," Physical Review D, vol. 68, no. 11, Article ID 094018, 2003. 
[27] B. S. Rajput, J.M.S. Rana, and H. C. Chandola, "Dyon dynamics in nonAbelian restricted gauge theory," Indian Journal of Pure and Applied Physics, vol. 26, p. 587, 1988.

[28] B. S. Rajput, S. Sah, and H. C. Chandola, "Confinement parameters in restricted chromodynamics," Il Nuovo Cimento A, vol. 106, no. 4, pp. 509-524, 1993.

[29] J. M. S. Rana, H. C. Chandola, and B. S. Rajput, "Supersymmetric dyons," Physical Review D, vol. 43, no. 10, pp. 3550-3556, 1991.

[30] B. S. Rajput, R. Bist, and H.C. Chandola, "Angular-momentum operators of non-Abelian dyons," Il Nuovo Cimento A, vol. 104, no. 5, pp. 697-705, 1991.

[31] B. S. Rajput, J. M. S. Rana, and H.C. Chandola, "Quark confinement through dyonic condensation," Progress of Theoretical Physics, vol. 82, pp. 153-161, 1989.

[32] B. S. Rajput, J. M. S. Rana, and H.C. Chandola, “Charge spectra of dyonic quarks," International Journal of Theoretical Physics, vol. 32, no. 2, pp. 357-371, 1993.

[33] Y. M. Cho, "Extended gauge theory and its mass spectrum," Physical Review D, vol. 23, no. 10, pp. 2415-2426, 1981.

[34] Y. M. Cho, "Glueball spectrum in extended quantum chromodynamics," Physical Review Letters, vol. 46, no. 5, pp. 302-306, 1981.

[35] Y. M. Cho, "Restricted gauge theory," Physical Review D, vol. 21, no. 4, pp. 1080-1088, 1980.

[36] B. S. Rajput, O. P. S. Negi, and J. M. S. Rana, "Magnetic symmetry and dyons," Pramana, vol. 31, no. 6, pp. 469-478, 1988.

[37] T. Suzuki, K. Ishiguro, Y. Mori, and T. Sekido, “Dual Meissner effect and magnetic displacement currents," Physical Review Letters, vol. 94, no. 13, Article ID 132001, 2005.

[38] A. Achucarro and T. Vachaspati, "Semilocal and electroweak strings," Physics Report, vol. 327, no. 6, p. 427, 2000.

[39] P. Forgács, S. Reuillon, and M. S. Volkov, "Twisted superconducting semilocal strings," Nuclear Physics B, vol. 751, no. 3, pp. 390-418, 2006.

[40] Y. M. Cho and P. G. O. Freund, "Non-abelian gauge fields as Nambu-Goldstone fields," Physical Review D, vol. 12, no. 6, pp. 1711-1720, 1975.

[41] Y. M. Cho and P. S. Jang, Physical Review D, vol. 12, p. 3189, 1975.

[42] M. K. Prasad and C. M. Sommerfield, "Exact classical solution for the 't Hooft monopole and the Julia-Zee dyon," Physical Review Letters, vol. 35, no. 12, pp. 760-762, 1975.

[43] P. Forgács and N. S. Manton, "Space-time symmetries in gauge theories," Communications in Mathematical Physics, vol. 72, no. 1, pp. 15-35, 1980.

[44] M. N. Chernodub, K. Ishiguro, Y. Mori et al., "Vacuum type of SU(2) gluodynamics in maximally Abelian and Landau gauges," Physical Review D, vol. 72, no. 7, Article ID 074505, pp. 1-14, 2005.

[45] V. G. Bornyakov, H. Ichie, Y. Koma et al., "Dynamics of monopoles and flux tubes in two-flavor dynamical QCD," Physical Review D, vol. 70, no. 7, Article ID 074511, 2004.

[46] V. G. Bornyakov, M. N. Chernodub, F. V. Gubarev et al., "Anatomy of the lattice magnetic monopoles," Physics Letters, vol. 537, no. 3-4, pp. 291-296, 2002. 

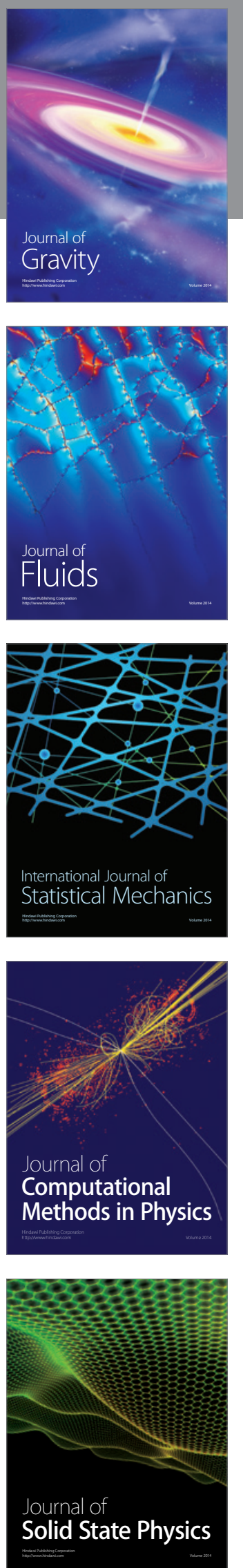

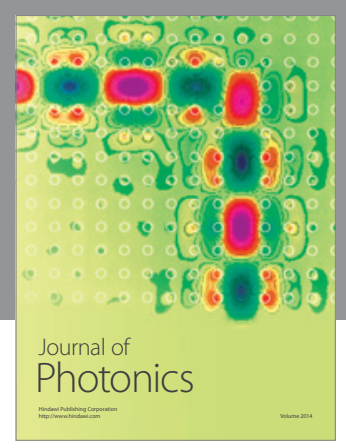

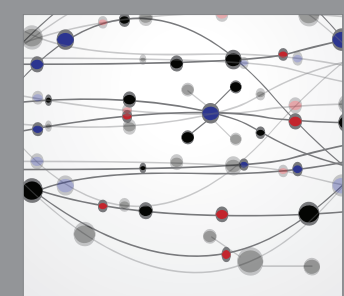

The Scientific World Journal
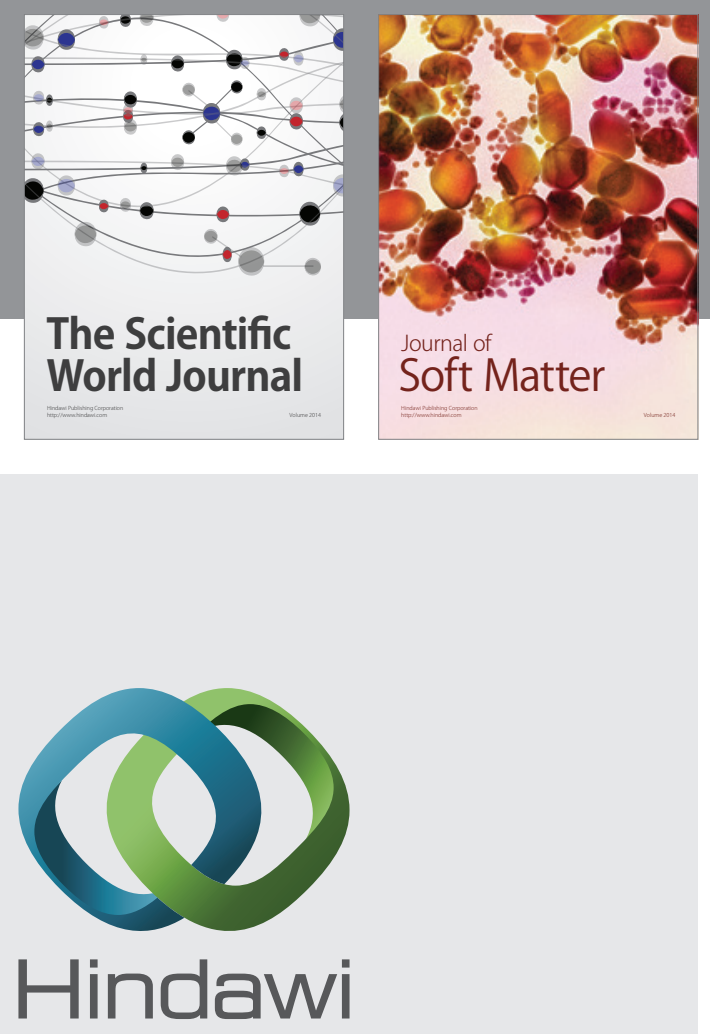

Submit your manuscripts at

http://www.hindawi.com
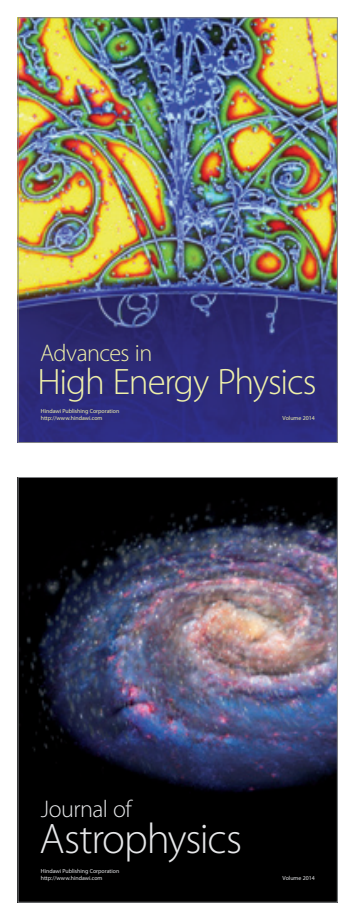
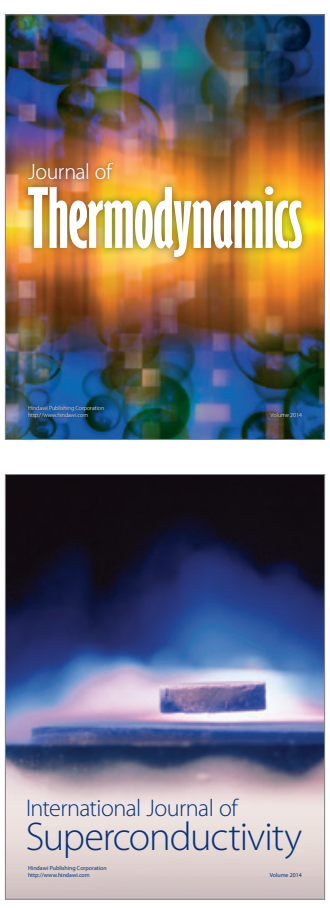
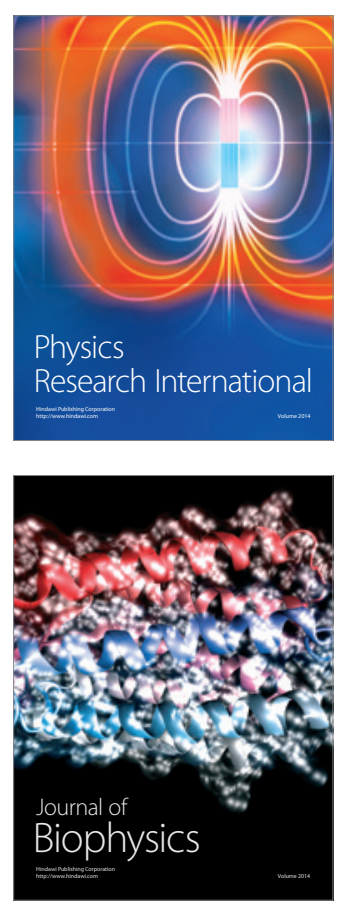
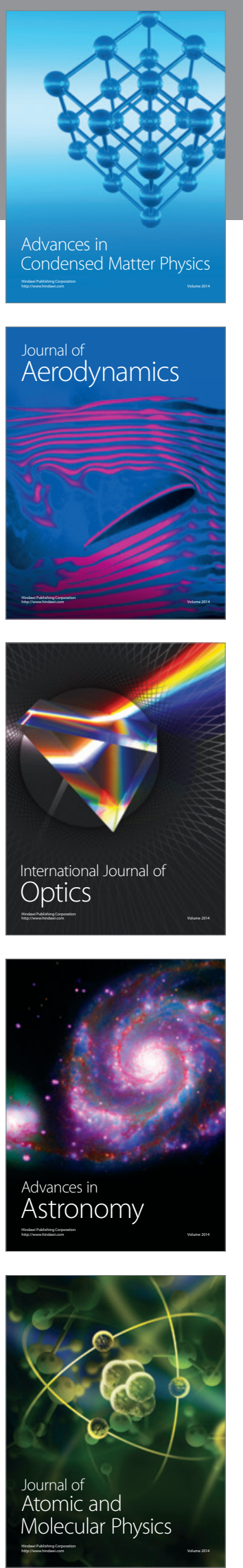